\title{
Research on Current Curative Expenditure and Influencing Factors Among Cardiovascular and Cerebrovascular Diseases Based on "System of Health Accounts 2011"
}

\section{Liwen Zhang}

Shihezi University School of Medicine

Xiaoju Li ( $\square$ lixiaoju2007@sina.cn )

Shihezi University School of Medicine https://orcid.org/0000-0003-2875-6646

Lu Mao

Shihezi University School of Medicine

Jielin Yang

Shihezi University School of Medicine

\section{Research}

Keywords: cardiovascular and cerebrovascular disease, current curative expenditure, influencing factors, SHA2011

Posted Date: September 13th, 2021

DOI: https://doi.org/10.21203/rs.3.rs-844590/v1

License: (c) (i) This work is licensed under a Creative Commons Attribution 4.0 International License.

Read Full License 
1 Research on Current Curative Expenditure and Influencing Factors among

2 Cardiovascular and Cerebrovascular Diseases Based on "System of Health

3 Accounts 2011"

$4 \quad$ Liwen Zhang ${ }^{1}$, Xiaoju $\mathrm{Li}^{1 *}$, Lu Mao ${ }^{1}$, Jielin Yang ${ }^{1}$

$5 \quad{ }^{1}$ Department of Public Health, Shihezi University School of Medicine, Shihezi, China.

6 Correspondence:

$7 \quad *$ Xiaoju Li

8 Department Of Public Health, Shihezi University School of Medicine, No.59, Bei 2 Road, Shehezi 9832000 , China.

10 Email: $\underline{\text { lixiaoju2007@ sina.cn }}$

11 Phone: +86-1890993834

12 
Abstract

Background: This study aims to research the total current curative expenditure (CCE) of cardiovascular and cerebrovascular diseases (CVDs) and their influencing factors in Xinjiang, China.

Methods: Through multistage stratified cluster sampling, the sample information of patients with CVDs in Xinjiang, in 2017, was collected. Under the framework of "System of Health Accounts 2011," the top-down allocation method was used to calculate the CCE of CVDs. Multiple linear regression was used to analyze the influencing factors.

Results: The CCE of CVDs in Xinjiang was 10.574 billion yuan; $86.81 \%$ of the CCE was spent in hospitals, of which $67.22 \%$ went to general hospitals. Coronary heart disease, hypertension, and cerebral infarction were the top three diseases among the treatment cost of CVDs, accounting for $74.20 \%$ of the total treatment cost. The CCE of older adults aged 65 years and above accounted for 43.51\%. The main factors affecting the hospitalization cost were length of stay, grade of the medical institution, operation, age, payment method, and gender.

Conclusions: CVDs consume numerous health funds in Xinjiang; prevention and control work focus on older adults. Further, the flow of treatment cost institutions is unreasonable; thus, the role of primary medical institutions in the prevention and treatment of chronic diseases should be strengthened. Reducing the length of hospital stay can effectively control the CCE.

Keywords: cardiovascular and cerebrovascular disease, current curative expenditure, influencing factors, SHA2011

\section{Background}

Cardiovascular and cerebrovascular diseases (CVDs) generally refer to the ischemic or 
viscosity, atherosclerosis, and hypertension [1]. Globally, CVDs have become a major public health

problem endangering human health. It is estimated that 17.8 million people died of CVDs in 2017, accounting for about one-third of global deaths [2]. More than 50\% of the deaths from chronic noncommunicable diseases are caused by CVDs, especially in middle-income and low-income countries with poor healthcare services; the disease burden situation is more severe [3]. In China, the mortality rate of cardiovascular disease ranked first in 2016, higher than that of cancer and other diseases [4]. At the same time, with the aging population and the progress of medical technology, the number of patients with all types of cardiovascular diseases showed an upward trend. From 1990-2016, the increase in the number of CVDs, excepting rheumatic heart disease, was relatively small (20.7\%); the increase of other types is more than 65\% [5]. Once diagnosed with CVDs, patients require long-term drug treatment and regular review, which leads to a substantial increase in treatment costs and places a huge economic burden on families. CVDs have become the main cause of the economic burden of chronic diseases.

Today, research on the treatment cost and influencing factors of CVDs is conducted globally. In the United States (U.S.), the direct cost of CVDs between 2013 and 2014 was estimated at $\$ 199.2$ billion, accounting for $14 \%$ of the total national health expenditure [6]. In Switzerland, the medical expenditure on CVDs was 10.1 billion Swiss francs in 2011, accounting for $15.6 \%$ of the total medical expenditure, thereby being the most expensive diseases [7]. In Mexico, the direct economic burden of four diseases — hypertension, heart failure, myocardial infarction, and atrial fibrillationwas $\$ 11.2$ billion in 2015 , equivalent to $4 \%$ of the country's total medical costs [8]. In China, the high expenditure on CVDs also poses great challenges to the government, families, and patients. In 2016, the total cost of hospitalization for CVDs was 19.085 billion yuan (AMI), while it was 25.419 billion yuan for intracranial hemorrhage and 60.105 billion yuan for cerebral infarction, with an average annual growth rate of $29.15 \%, 16.88 \%$, and $22.24 \%$, respectively, since 2004 [9]. However, most studies have focused primarily on economic analysis of certain types of CVDs with high incidence and serious illness. There are fewer studies on the treatment cost of cardiovascular and 
cerebrovascular diseases in a large sample.

Xinjiang is one of the major provinces in Western China and incurs a heavy burden from CVDs. In 2017, the mortality rates of heart disease in urban and rural areas were 95.40/100000 and 136.97/100000, respectively, and heart disease ranked first among the causes of death in Xinjiang. Therefore, it is necessary to study the treatment cost of CVDs in Xinjiang Province. The System of Health Accounts 2011 (SHA2011) is a new method of total health expenditure accounting introduced by the OECD, Eurostat, and the WHO in 2011. This system is an internationally recognized monitoring and analysis tool for health financing systems, which has good international and regional comparability. Using this method to calculate the total health cost of CVDs can provide important data support for the government to accurately formulate targeted prevention and control strategies for CVDs and to determine a reasonable funding plan. In this study, using SHA2011, comprehensive accounting and analysis of the total cost of treatment of CVDs were conducted in Xinjiang in 2017 from the perspective of service providers and population benefits. Furthermore, we analyzed the survey data to determine the influencing factors of the treatment burden of patients with CVDs.

\section{Methods and analysis}

\section{Macro data sources}

The macro data of this study come from the "2017 Xinjiang health financial annual report," the "2017 health statistics annual report," and the government health investment monitoring system of Xinjiang Uygur Autonomous Region; and the total amount of recurrent health expenditure of the whole province is summarized.

\section{Case data field investigation}

In this study, a multistage stratified sampling method was used to select medical and health institutions as the monitoring points of total health expenditure. In the first stage, five regions were selected according to the actual situation of the autonomous region, including Urumqi, the Bayinguoleng Mongolian Autonomous Prefecture, Ili, Hami, and Kashgar. In the second stage, three 
to five counties (districts) were selected according to the working foundation and information construction of health and family planning medical institutions. Five streets, communities, and townships were chosen in the selected districts and counties, and one to three villages or neighborhood committees were selected as samples in each township. After determining the survey area, sampling was conducted according to the level and classification of health institutions. Three hundred and fifty-two health institutions were investigated. The CVD cases collected numbered 711,116 , including 539,293 outpatient cases and 171,823 inpatient cases. The basic information of patients includes age, gender, disease, cost, length of hospital stay, and financing plan.

\section{Accounting method}

SHA2011 adopts the top-down accounting method, that is, after the regular health expenses are obtained from the macro data, the allocation coefficient obtained from the field survey data are used to decompose the regular health expenses into other dimensions for accounting and the current curative expenditure (CCE) of CVDs in the whole province.

The CCE funds come from medical income, government project subsidies, and basic expenditure subsidies (including outpatient and inpatient). Taking the hospital outpatient CCE as an example ( $S_{O C C E}$ ), the hospital outpatient medical income $\left(S_{O M I}\right)$ is the product of the hospital's total outpatient income $\left(S_{T O I}\right)$ and sharing coefficient $\alpha$. The hospital total outpatient income is obtained by summarizing the relevant data from the Xinjiang Health Statistics Yearbook 2018 and Xinjiang Health

Finance Annual report 2018; the coefficient $\alpha$ is the proportion after deducting the preventive

outpatient income $\left(S_{P O I}\right)$. The outpatient basic expenditure subsidy $\left({ }{ }_{O B S}\right)$ is the result of the allocation of basic expenditure subsidies in treatment services, and the outpatient project subsidy ( $S_{O P S}$ ) refers to the outpatient service projects assigned to the hospital. The specific accounting 
method is as follows :

$$
S_{O C C E}=S_{O M I}+S_{O B S}+S_{O P S}
$$

$$
S_{O M I}=S_{T O I} \times \alpha
$$

$$
\alpha=1-\frac{S_{P O I}}{S_{T O I}}
$$

Outpatient basic curative expenditure subsidy:

$$
S_{O B S}=S_{C B S} \times(1-\beta)
$$

117 Where $S_{C B S}$ represents the curative basic expenditure subsidy, ${ }^{d}$ represents the total number of

inpatient bed days, and ${ }^{e_{i}}$ represents the number of outpatient curative patients. $\mathrm{k}=0.1$ and hospital bed day.

$$
S_{C B S}=S_{T B S} \times \gamma
$$

$$
e_{i}=f_{i} \times \gamma
$$

$$
\beta=d_{i} /\left(d_{i}+e_{i}^{124} \times\right)
$$

$125 f_{i}$ represents the total number of outpatient visits, ${ }^{g_{i}}$ represents the number of preventive outpatient

126 visits of sample institutions, and $h_{i}$ represents the total number of outpatient visits of sample 
institutions. The proportion of preventive outpatient income in the total income and the proportion of preventive outpatient visits in the total outpatient visits are all derived from the data of the sample institutions.

\section{Analysis of influencing factors of hospitalization expenses of CVDs}

The main factors influencing hospitalization expenses of CVDs were determined through the analysis of hospitalization expenses. Spearman correlation analysis was used to test the correlation among hospitalization expenses whether surgery, length of stay, insurance status, institution level, admission season, gender, or age. Since the length of hospital stay and the total cost were positively skewed to the peak distribution, the logarithmic transformation was performed before the analysis. Multiple linear regression was used to analyze the influencing factors. Test level $\alpha=0.05$. Stata 12.0 and SPSS 26.0 were used to analyze the data.

\section{Results}

\section{A fundamental result in the CCE of CVDs}

In 2017, the treatment cost of CVDs in Xinjiang was 10.574 billion yuan, accounting for $28.69 \%$ of the treatment cost of chronic non-communicable diseases (36.862 billion yuan) and $1.24 \%$ of the GDP. The hospitalization cost was 7.412 billion yuan, accounting for $70.09 \%$, and the outpatient cost was 3.163 billion yuan, accounting for $29.91 \%$.

\section{Institutional distribution of the CCE for CVDs}

The CCE of CVDs was spent mainly in hospitals, accounting for $86.81 \%$ (67.22\% in general hospitals, $16.28 \%$ in traditional Chinese medicine hospitals, and $3.31 \%$ in specialized hospitals); the proportion of primary medical and health institutions was $10.71 \%$. Among the total CCE, $74.12 \%$ went to inpatient services, while only $29.91 \%$ went to outpatient services (Table 1 ).

[Place tab 1 here]

\section{CCE of different CVDs}


In the CCE of CVDs, the top three were coronary heart disease, hypertension, and cerebral infarction, accounting for $74.20 \%$ of the total CCE. From the perspective of disease service function distribution, the CCE of hypertension was mainly outpatient service, accounting for more than half of the outpatient CCE. In the inpatient service, cardiovascular diseases accounted for nearly $70 \%$, and cerebrovascular diseases accounted for $23.55 \%$ (Figure 1).

[Place fig 1 here]

\section{Distribution of beneficiaries of the CCE of CVDs}

According to the age distribution of the population, the CCE of CVDs in 2017 was concentrated in middle-aged and elderly people aged $45-84$ years, with a CCE of 9.096 billion yuan, accounting for $86.02 \%$. It is worth noting that this was spent by $43.51 \%$ of elderly people aged 65 years and above, while the proportion of the population they accounted for was only $6.48 \%$, and the proportion of CCE was significantly higher than that of the population. Moreover, inpatient service was the main group for CVDs in all age groups, and the proportion of inpatient service cost was more than $60 \%$ (Figure 2).

[Place fig 2 here]

The disease composition of the CCE of CVDs differed across age groups. Taking the results of 2017 as an example, the proportion of coronary heart disease and essential hypertension in all age groups above 35 years old was significantly higher than that of other diseases. Among them, the age group with the highest treatment cost for primary hypertension was 35-79 years old, accounting for more than $30 \%$. The treatment cost for coronary heart disease ranks first, after the age of 60 , as this is the main disease endangering the health of the older adults group (Figure 3).

[Place fig 3 here]

\section{Factors influencing the CCE of hospitalization for CVDs}



between hospitalization expenses and age $(r=0.115, \mathrm{P}<0.001)$, gender $(\mathrm{r}=0.058, \mathrm{P}<0.001)$, length of stay $(\mathrm{r}=0.564, \mathrm{P}<0.001)$, type of insurance $(\mathrm{r}=0.362, \mathrm{P}<0.001)$, institution level $(\mathrm{r}=0.582, \mathrm{P}<$ $0.001)$ and surgery $(\mathrm{r}=0.232, \mathrm{P}<0.001)$. There was a negative correlation between age and gender, insurance type and surgery (Table 2). regression equation was established by multiple linear regression analysis, the equation was statistically significant $(\mathrm{F}=20873.31, \mathrm{P}<0.001)$; and the diagnosis of multicollinearity was all within

the normal range without the presence of collinearity. From the standard partial regression coefficients, the top five factors affecting hospitalization CCE were whether the institutions were county- (District-) level institutions, days of hospitalization, whether the institutions were municipallevel institutions, whether patients were older than 65 years, and whether or not patients had surgery. All independent variables included in the regression equations contributed $52.2 \%$ to the variability of the dependent variable (Tables 3).

[Place tab 3 here]

\section{Discussion}

CVDs have become a major health problem in China. It is reported that the age-standardized prevalence rate of cardiovascular disease in China increased by $14.7 \%$ from 1990-2016 [10]. At the same time, the total hospitalization expenses of CVDs are also increasing rapidly. Since 2004, the average annual growth rate has exceeded $20 \%$, far higher than the growth rate of the GDP [9]. More patients with cardiovascular disease spend money on medical care and treatment, which causes a serious economic burden. In this study, it was noted that the treatment cost of CVDs in Xinjiang in 2017, reached 10.574 billion yuan, accounting for $20.37 \%$ of the treatment cost of all diseases (51.916 billion yuan), higher than the national average of $18.3 \%$ [1]. This shows that CVDs consume 
numerous medical and health resources. Therefore, from the perspective of reducing the economic burden of disease, CVDs should be the focus of disease prevention and control in Xinjiang in the future.

It is worth noting that older adults have become the main victims of CVDs, which is an important factor influencing the growth of health costs. The regression analysis also confirmed that the impact of adults over 65 years old on health expenditure is significantly greater than that of other age groups. The treatment cost of this group accounted for $43.51 \%$ of the total treatment cost of CVDs. In the past three population censuses, the proportion of adults over 65 years old in Xinjiang reached $3.91 \%$, $4.67 \%$, and $6.48 \%$, respectively, showing an obvious acceleration and the serious aging problem. Due to the continuous deterioration of physical functions, older adults often suffer from a variety of complications concurrently, which undoubtedly creates a huge economic burden on older adults whose income source is primarily family support [11]. It can be seen that strengthening the intervention of risk factors for older adults and realizing healthy aging is the inevitable way to cope with the changes in the population structure in Xinjiang.

In this study, it was noted that the top three diseases with the highest treatment cost were coronary heart disease, hypertension, and cerebral infarction, with a total amount of 7.846 billion yuan, accounting for three-quarters of the total cost of treatment of CVDs. Research should focus on targeted prevention and control for these diseases. Studies have noted that more than $80 \%$ of the incidence of ischemic cardiovascular disease can be attributed to dyslipidemia, hypertension, and diabetes and $20 \%$ to lifestyle and other factors [12]. Therefore, the prevention and control of CVDs, should focus on primary prevention by forming healthy eating habits and behavior. Further, the outpatient control of hypertension, hyperlipidemia, and hyperglycemia according to the risk factors of the disease to effectively prevent the occurrence of cardiovascular and cerebrovascular events should be another focal point. At present, standardized management of hypertension and hyperglycemia has been conducted steadily in Xinjiang. From 2011-2019, the standardized management of these two chronic diseases in basic public health service projects increased by $212 \%$ and $181 \%$, 
respectively [13]. However, the prevention and control of dyslipidemia still lag behind hypertension and diabetes. International studies have shown that for every $1 \mathrm{mmol} / \mathrm{L}$ decrease in LDL cholesterol, the risk of cardiovascular disease decreased by $22 \%$ [14]. Therefore, strengthening the outpatient control of dyslipidemia can effectively improve the cost-effectiveness of secondary prevention.

From the perspective of institutional flow, the cost of CVD treatment in Xinjiang is concentrated in hospitals, accounting for $86.81 \%$, and the proportion of primary medical institutions was low. There is an "inverted triangle" phenomenon in the health resource allocation of the treatment cost of CVDs, which is not consistent with the planning structure of chronic disease prevention and control strategy and hierarchical diagnosis and treatment policy. We should further implement the hierarchical diagnosis and treatment policy, improve the health service ability of grassroots institutions, and realize the reasonable diversion of medical treatment.

The hospitalization expenses of patients with CVDs accounted for $70.09 \%$ of the treatment expenses in Xinjiang. The rapid growth of inpatient services is the main driving force of the growth of medical expenses [15]. According to the regression analysis of the influencing factors of hospitalization expenses, the length of stay is the most important factor, which is consistent with other research results [16]. Logically, the longer the length of hospital stay, the more medical resources will be consumed, which leads to higher hospital costs [17]. Shortening hospital stays is the most direct and effective way to reduce the total health cost of patients with CVDs. The level of medical institutions is another key factor affecting hospitalization expenses. In this study, it was noted that the county- (District-) level medical institutions are the protective factor of hospitalization expenses, which shows that lower-level medical institutions can effectively reduce the burden of disease costs of residents $[18,19]$. However, some studies have shown that with the advancement of healthcare reform, grassroots medical and health institutions are still facing many problems, such as a lack of human resources, insufficient utilization of services, and low service capacity [20]. The allocation of health resources in Xinjiang shows that the Gini coefficient of the geographical distribution of medical and health resources in Xinjiang in 2016 is 0.5572 , which is uneven in geographical 
distribution, especially in remote grassroots areas, where residents have poor access to health services [21]. Combined with the flow direction of hospitalization expenses, we should further improve the supply quantity, the quality of healthy human resources, and the backward situation of medical and health services in grassroots and county-level medical institutions. The impact of surgery on hospitalization cost is second only to the length of stay and the level of medical institutions. Surgeries will not only increase the cost but also extend the length of the hospital stay [22]. In the study sample, the average treatment cost of surgical patients was 3.18 times that of non-surgical patients. The treatment cost of surgical patients is significantly higher than that of non-surgical patients because cardiovascular surgery requires expensive stents and imported drugs. In this study, it was also confirmed that the cost of medical insurance patients is higher than that of self-funded patients. Wang Ting and other scholars found that the gradual improvement of the social basic medical security system could lead to an increase in the total cost of healthcare [23]. It can be seen that the gradual improvement of the medical security system not only promotes patients' ability to obtain better medical services but also increases the total medical expenses.

\section{Conclusion}

The research on the CCE of patients with CVDs in Xinjiang province shows that the economic burden of patients with CVDs is relatively heavy. The top three influencing factors of hospitalization expenses were length of stay, grade of medical institution, and whether or not surgery was performed. It is suggested to promote the hierarchical diagnosis and treatment system, strengthen the role of primary medical institutions in the prevention and treatment of chronic diseases, focus on monitoring middle-aged and elderly people, and effectively control the CCE by reducing the length of hospital stays.

\section{List of abbreviations}

CVDs: Cardiovascular and cerebrovascular diseases

CCE: current curative expenditure 


\section{Declarations}

Ethics approval and consent to participate

281

The study was supported by Health Economics Association of Xinjiang Uygur Autonomous Region and Ethics Committee of Shihezi University and they claimed that they approved this study. All procedures performed in studies involving human participants were in accordance with the ethical standards of the institutional and national research committee and with the Helsinki declaration and its later amendments or compa-rable ethical standards. All the informed consent form and the data we used have been informed to Ethics Committee of Shihezi University and got their permission.

\section{Consent for publication}

All of the data is allowed by patients and medical institutions.

\section{Availability of data and materials}

The datasets generated and/or analyzed during the current study are available from the corresponding author on reasonable request.

\section{Competing interests}

The authors declare that they have no competing interests.

\section{Funding}

This work was supported by the Ministry of Education in China Project of Humanities and Social Sciences- Xinjiang (Project No.18XJJCZH001) and Shihezi University high level talents research project (Project No.RCZK201907).

\section{Author's Contributions}

LZ had full access to all of the study and takes responsibility for the integrity of the data and the accuracy of the data analysis; XL and LM was responsible for concept and design; XL and JY assisted in revision of the manuscript. All authors read and approved the final manuscript.

\section{Acknowledgments}

The authors express thanks to China National Health Development Research Center for their help with SHA 2011 analysis, and also gratefully acknowledge the support of the Health Commission of Xinjiang Uygur Autonomous Region. Thanks to Xinjiang's Department of Finance, Civil Affairs, and other departments to provide basic accounting data, and thanks for the data support provided by the sampled 
medical and health institutions.

\section{References}

1. ZHANG Yu-hui ZTCP: Study on Accounting and Projection of Curative Expenditure on Cardiovascular and Cerebrovascular Diseases in China. Chinese Health Economics 2019, 38(05):18-22.

2. Collaborators GDAI: Global, regional, and national age-sex-specific mortality for 282 causes of death in 195 countries and territories, 1980-2017: a systematic analysis for the Global Burden of Disease Study 2017. LANCET 2018, 392(10159):1736-1788.

3. Collaborators NC: NCD Countdown 2030: worldwide trends in non-communicable disease mortality and progress towards Sustainable Development Goal target 3.4. LANCET 2018, 392(10152):10721088 .

4. HU Shengshou GRLL: Summary of the 2018 Report on Cardiovascular Diseases in China. Chinese Circulation Journal 2019, 34(03):209-220.

5. LI Yichong LSZX: Report on Burden of Cardiovascular Diseases From 1990 to 2016 in China. Chinese Circulation Journal 2019, 34(08):729-740.

6. Benjamin EJ, Virani SS, Callaway CW, Chamberlain AM, Chang AR, Cheng S, Chiuve SE, Cushman M, Delling FN, Deo R et al: Heart Disease and Stroke Statistics-2018 Update: A Report From the American Heart Association. CIRCULATION 2018, 137(12):e67-e492.

7. Wieser S, Riguzzi M, Pletscher M, Huber CA, Telser H, Schwenkglenks M: How much does the treatment of each major disease cost? A decomposition of Swiss National Health Accounts. The European journal of health economics 2018, 19(8):1149-1161.

8. Stevens B, Pezzullo L, Verdian L, Tomlinson J, Estrada-Aguilar C, George A, Verdejo-Paris J: The economic burden of hypertension, heart failure, myocardial infarction, and atrial fibrillation in Mexico. Arch Cardiol Mex 2018, 88(3):241-244.

9. CHEN Weiwei GRLL: Summary of the 2017 Report on Cardiovascular Diseases in China. Chinese Circulation Journal 2018, 33(01):1-8.

10. Liu S, Li Y, Zeng X, Wang H, Yin P, Wang L, Liu Y, Liu J, Qi J, Ran S et al: Burden of Cardiovascular Diseases in China, 1990-2016: Findings From the 2016 Global Burden of Disease Study. JAMA CARDIOL 2019, 4(4):342-352.

11. Yanhui JXZ: Analysis on the Main Source of Livelihood and Economic Security of the Chinese Elderly. POPULATION JOURNAL 2013, 35(02):42-48.

12. Beifan Z: From epidemiological research to clinical prevention and treatment practice. Chinese Journal of Cardiology 2003(12):14-15.

13. ZHANG Yi ZWZJ: Review of prevention and control of chronic non communicable diseases and public health in Xinjiang. Disease prevention and control Bulletin 2020, 35(3):81-85.

14. Baigent C, Blackwell L, Emberson J, Holland LE, Reith C, Bhala N, Peto R, Barnes EH, Keech A, Simes $\mathrm{J}$ et al: Efficacy and safety of more intensive lowering of LDL cholesterol: a meta-analysis of data from 170000 participants in 26 randomised trials. The Lancet 2010, 376(9753):1670-1681.

15. Zhai T, Goss J, Dmytraczenko T, Zhang Y, Li J, Chai P: China's Health Expenditure Projections To 2035: Future Trajectory And The Estimated Impact Of Reforms. Health Aff (Millwood) 2019, 38(5):835843.

16. Zhu Y, Liu C, Zhang L, Fang Q, Zang S, Wang X: How to control the economic burden of treating 
cardio-cerebrovascular diseases in China? Assessment based on System of Health Accounts 2011. J GLOB HEALTH 2020, 10(1):10802.

17. He J, Yin Z, Duan W, Wang Y, Wang X: Factors of hospitalization expenditure of the genitourinary system diseases in the aged based on "System of Health Account 2011" and neural network model. $J$ GLOB HEALTH 2018, 8(2):20504.

18. LI Yanjun WLYC: Research on the Restrictive Factors and Countermeasures of the Development of Primary Health Care Institutions ——-Based on the Grounded Theory. Health Economics Research 2019, 36(03):49-52.

19. WANG Zhen WCHX: Analysis of the Inpatient Flow and Hospital Cost Among New Rural Cooperative Medical System in Gansu. Chinese Health Economics 2017, 36(02):33-35.

20. Yapeng Y: Problems and Countermeasures in comprehensive reform of primary medical and health institutions. WESTERN FINANCE AND ACCOUNTING 2014(2):68-71.

21. ZHANG Ye ZCWZ: $R$ esearch on the Equity and Influencing Factors of Health $R$ esource Allocation in Xinjiang from 2004 to 2016: A Comprehensive Perspective Based on "Population Fairness" and “Geographical Equality” . he Chinese Health Service Management 2019, 36(07):499-509.

22. Kusachi S, Kashimura N, Konishi T, Shimizu J, Kusunoki M, Oka M, Wakatsuki T, Kobayashi J, Sawa Y, Imoto H et al: Length of Stay and Cost for Surgical Site Infection after Abdominal and Cardiac Surgery in Japanese Hospitals: Multi-Center Surveillance. SURG INFECT 2012, 13(4):257-265.

23. Xin W: Dynamic relationship between social basic medical security and total health expenditure. Chinese Journal of Health Statistics 2013, 30(01):77-79.

\section{Figures}

Table 1 institutional distribution of curative care expenditure of CVDs in Xinjiang in $2017^{*}$

\begin{tabular}{|c|c|c|c|c|c|c|}
\hline & \multicolumn{2}{|c|}{ Outpatient } & \multicolumn{2}{|l|}{ Inpatient } & \multicolumn{2}{|l|}{ Total } \\
\hline & (million) & $\%$ & (million) & $\%$ & (million) & $\%$ \\
\hline Hospital & 2533.54 & 27.60 & 6645.78 & 72.40 & 9179.33 & 86.81 \\
\hline General hospital & 1987.20 & 27.96 & 5120.29 & 72.04 & 7107.49 & 67.22 \\
\hline Traditional Chinese medic & 1479.39 & 27.84 & 1242.51 & 72.16 & 1721.90 & 16.28 \\
\hline Special hospital & 66.95 & 19.13 & 282.99 & 80.87 & 349.94 & 3.31 \\
\hline Primary medical institution & 367.30 & 32.42 & 765.72 & 67.58 & 1133.00 & 10.71 \\
\hline
\end{tabular}




$\begin{array}{llllllr}\text { Outpatient service institution } & 261.63 & 100.00 & 0.00 & 0.00 & 261.63 & 2.47 \\ \text { Public health institution } & 0.05 & 100.00 & 0.00 & 0.00 & 0.05 & 0.00 \\ \text { Total } & 3162.53 & 29.91 & 7411.50 & 70.09 & 10574.01 & 100.00\end{array}$

372 *Primary medical institution include township health centers and community health centers; Outpatient service institution

373 settings include village health rooms and health service stations; Public health institution includes disease control, maternity

374 and child care specialist hospitals, etc.

375

Tables 2 Variable correlation analysis $(\mathrm{r})^{*}$

\begin{tabular}{|c|c|c|c|c|c|c|c|c|}
\hline & & 1 & 2 & 3 & 4 & 5 & 6 & 7 \\
\hline 1 & Hospitalization expenses & 1 & & & & & & \\
\hline 2 & Age & $.115 * *$ & 1 & & & & & \\
\hline 3 & Sex & $.058 * *$ & $-.023 * *$ & 1 & & & & \\
\hline 4 & Length of stay & $.564 * *$ & $.061 * *$ & $.012 * *$ & 1 & & & \\
\hline 5 & Insurance & $.362 * *$ & $-.026 * *$ & $.055 * *$ & $.247 * *$ & 1 & & \\
\hline 6 & Institution level & $.582 * *$ & 0.005 & $.071 * *$ & $.305 * *$ & $.486 * *$ & 1 & \\
\hline 7 & Surgery & $.232 * *$ & $-.127 * *$ & $.019 * *$ & $.114^{* *}$ & $.060 * *$ & $.120 * *$ & 1 \\
\hline
\end{tabular}

377 *Abbreviations: $\mathrm{r}=$ Spearman's correlation coefficient. ${ }^{*} \mathrm{p}<0.05, * * \mathrm{p}<0.01$. For insurance: urban employees' basic medical

378 insurance $=4$, urban residents' basic medical insurance $=3$, new rural cooperative medical care $=2$, self-funded $=1$. For institution

379 level: provincial institution=3, municipal institution=2, district and county institution=1. For surgery: no surgery=0 and surgery $=1$. For sex: female $=1$ and male $=2$.

381

Table 3 Influencing factors regression analysis of hospitalization CCE of CVDs in Xinjiang*

\begin{tabular}{|c|c|c|c|c|c|}
\hline \multirow[t]{2}{*}{ Model } & \multicolumn{2}{|c|}{$\begin{array}{l}\text { Unstandardized } \\
\text { Coefficients }\end{array}$} & \multirow{2}{*}{$\begin{array}{l}\text { Standardized } \\
\text { coefficients } \\
\text { Beta }\end{array}$} & \multirow[t]{2}{*}{$\mathrm{t}$} & \multirow[t]{2}{*}{ Sig. } \\
\hline & $\overline{\mathrm{B}}$ & Std. Error & & & \\
\hline Constant & 3.549 & 0.004 & & 988.722 & $<0.001$ \\
\hline Age & 0.003 & 0.000 & 0.122 & 71.194 & \\
\hline Sex & 0.021 & 0.001 & 0.029 & 17.201 & $<0.001$ \\
\hline
\end{tabular}


Insurance

Self-paying
new rural cooperative medical care

$\begin{array}{llll}-0.079 & 0.003 & -0.058 & -29.903 \quad<0.001\end{array}$

urban residents' basic medical insurance

$-0.026$

0.002

$-0.034$

$-14.517$

$<0.001$

Institution level

District and county institution

$\begin{array}{lllll}-0.380 & 0.002 & -0.509 & -204.493 & <0.001 \\ -0.199 & 0.002 & -0.233 & -104.783 & <0.001 \\ 0.227 & 0.002 & 0.172 & 100.792 & <0.001\end{array}$

"Note: Setting dummy variables for insurance type and medical institution level. For Institution level: district and county

384 institution="1" if it was and "0" otherwise; municipal institution="1" if it was and "0" otherwise. As for the provincial institution, setting to "0". For insurance: Self-paying="1" if it was and "0" otherwise; new rural cooperative medical car="1" if it was and "0" otherwise; urban residents' basic medical insurance="1" if it was and "0" otherwise. As for urban employees' basic medical insurance, setting to "0". 